\title{
Riesgos virales en odontoestomatología. Campaña de vacunación frente a hepatitis
}

\author{
de Juanes JR*, Arrazola MP**, de Juanes A***, Lago E**** \\ Rocha $M^{* * * *}$, Gil $\mathrm{P}^{* * * *}$, Bascones $\mathrm{A}^{* * * * * *}$
}

\section{RESUMEN}

Se han descrito los principales riesgos biológicos en la práctica odontoestomatológica así como las precauciones estándar que deben tenerse presentes.

Se presenta la Campaña de Vacunación frente a las hepatitis A y B ofertada por un Colegio de Odontólogos y Estomatólogos con los datos de aceptación de la misma.

Entre los candidatos a vacunarse frente a la hepatitis B, el $69 \%$ de los odontólogos, el 59\% de los estomatólogos y el $63 \%$ de los ayudantes completaron la pauta vacunal.

La vacunación frente a hepatitis en la práctica odontoestomatológica es la forma más contundente de evitar enfermedades no deseadas.

Palabras clave: Riesgos virales, Precauciones estándar, vacuna Hepatitis B, odontólogos

\begin{abstract}
To describe and review the main biological risks in dental practice and the standard precautions to be adopted by dentists and stomatologists.

A vaccination campaign against hepatitis $A$ and $B$ organised by the Madrid College of Dentists and stomalogists is presented along with data on its acceptance.

Of the 197 dentists candidates for hepatitis B vaccination, 69\% underwent the complete vaccination program; Of the 85 stomatologists candidates for hepatitis B vaccination, 59\% completed the vaccination program. $63.3 \%$ of the dental assistants, hygienists, prothetists and registered nurses completed the vaccination program. We can consider this hepatitis B as the most important risk for dentists and stomatologists. The Madrid College of Dentists and Stomatologists was especially aware, when it organised this campaign, of the need to protect colleagues at the beginning of their professional life.
\end{abstract}

Key words: Virus risk, Standard Precautions, Hepatitis B vaccination, dentist.

* Jefe de Servicio de Medicina Preventiva.

** Médico Adjunto de Medicina Preventiva.

*** Médico Residente.

**** DUE. Hospital Universitario "12 de Octubre". Madrid.

***** Catedrático de Odontología. Universidad Complutense de Madrid.

de Juanes JR, Arrazola MP, de Juanes A, Lago E, Rocha M, Gil P, Bascones A. Riesgos virales en odontoestomatología. Campaña de vacunación frente a hepatitis. Av. Odontoestomatol 2003; 19-1: 21-28 


\section{INTRODUCCIÓN}

El personal que trabaja en las distintas áreas sanitarias eatá sometido a diversos riesgos biológicos, siendo los virus los agentes más importantes a los que están expuestos.

Un importante grupo de sanitarios es el formado por los colectivos de estomatólogos, odontólogos y sus colaboradores, como higienistas, protésicos, enfermeras o auxiliares (GOUPIL, 1995).

Los estomatólogos y odontólogos se pueden exponer a riesgos biológicos a través de accidentes percutáneos o cutáneo mucosos que supongan la exposición a la sangre de los pacientes; como consecuencia, los afectados pueden terminar desarrollando lesiones orgánicas de importante trascendencia (REICHART, 1994; DE JUANES, 1996).

El riesgo de transmisión biológica desde un paciente infectado a un sanitario está ampliamente descrito. (CDC, 1993; YOUNAI, 2001)

Comentaremos someramente los principales riesgos biológicos en odontoestomatología para, a continuación, proceder a la recomendación general de una estrategia de vacunación frente a los virus de hepatitis B y hepatitis A cuando sea posible:

1. Agentes biológicos implicados

2. Problemática paciente - sanitario

3. Prevención: Principios generales en el trabajo

4. Normas en la práctica clínica

5. Estrategias de prevención: campaña de vacunación

\section{AGENTES BIOLÓGICOS IMPLICADOS}

La mayoría de los agentes biológicos que conllevan un riesgo en odontoestomatología corresponden, principalmente, a microorganismos que se encuentran en sangre y secreciones orales (CDC 1993b; PALENIK, 1999). Los virus más frecuentemente implicados son el virus de la hepatitis B (VHB) y el virus de la hepatitis $\mathrm{C}(\mathrm{VHC})$, no existiendo prácticamente riesgo con el virus de la inmunodeficiencia humana $(\mathrm{VIH})$. El virus de la hepatitis $\mathrm{A}$, como riesgo de transmisión feco-oral, se podría encontrar en áreas de endemicidad alta y poca higiene. (ASHKENAZI, 2001). Menor trascendencia tienen otros virus, como el herpesvirus o el virus de la hepatitis G (VHG). (GILLCRIST, 1999).

El virus de la hepatitis B (VHB) afecta a más de 350 millones de personas en el mundo. España está en una zona de endemicidad media, pudiendo asumir la existencia de entre 300 y 500.000 portadores (DE JUANES, 1994). Anualmente es responsable, de forma directa o indirecta, de numerosas muertes en el mundo (ALTER, 1990; HOOFNAGLE, 1990; CIESIELSKI, 1992).

El virus de la hepatitis C (VHC) afecta a millones de personas en el mundo. En España, se estima que el número de afectados está entre 300 y 800.000 personas (RESNICK, 1993); frecuencia permanece asintomático. Se acepta una cronificación del 40-60\% de los casos (SALLIE, 1994). La principal vía de transmisión es a través de sangre contaminada y aunque el virus puede encontrarse en saliva, esta vía de transmisión es muy remota (CHEN, 1995; CLEVELAND, 1999).

El virus de la inmunodeficiencia humana (VIH) es responsable de un incremento continuo de casos de SIDA. Los problemas que plantea el VIH en el ámbito odontoestomatológico están relacionados con la posibilidad de contacto con sangre de pacientes infectados, siendo el riesgo de transmisión prácticamente nulo (CDC, 1991). En todo caso si se produjera un accidente habrá que valorar la necesidad de profilaxis postexposición (Ministerio de Sanidad y Consumo, 2001).

El virus de la hepatitis A no es un riesgo directo para el sanitario pero puede considerarse un riesgo colateral en función de la endemicidad del virus en el área donde se trabaje.

\section{PROBLEMÁTICA PACIENTE-SANITARIO}

La transmisión viral desde un paciente infectado al sanitario es mucho más importante que en sentido contrario, al menos si lo consideramos desde el punto de vista numérico. 
El problema deriva de la accidentabilidad del personal sanitario en general y del odontoestomatólogo en particular. (WOLF, 1992; CLEVELAND, 1994).

\section{PREVENCIÓN: PRINCIPIOS GENERALES EN EL TRABAJO}

Todo estomatólogo, odontólogo y sus ayudantes, deben tener presentes permanentemente los siguientes principios generales para evitar el posible riesgo de infección derivado de la práctica asistencial:

- Los agentes biológicos comentados (virales) se transmiten en el medio laboral a través de la sangre y/o ciertos fluidos.

- Toda sangre es potencialmente infecciosa.

- No se conocen todos los pacientes portadores de virus.

- El contacto con la sangre se produce a través de accidentes.

- En todo momento hay que tener en cuenta las normas elementales en cuanto a ropa de trabajo, material adecuadamente desinfectado o esterilizado, precauciones y prohibiciones en el área de trabajo cotidiano y conducta a seguir en caso de accidente.

\section{NORMAS EN LA PRÁCTICA CLÍNICA}

El conjunto de normas y recomendaciones que se deben tener presentes en el trabajo diario, conocidas como PRECAUCIONES UNIVERSALES (CDC, 1988), se pueden agrupar en vacunación contra la hepatitis B y controles de ingeniería en las prácticas de trabajo.

\section{- Vacunación frente a hepatitis B}

Todos los estomatólogos, odontólogos y su personal colaborador deben estar adecuadamente inmunizados contra la hepatitis B. (CLEVELAND, 1996; VIGNARAJAH, 1998). Todos los profesionales en activo que no lo estén, deben vacunarse.

Para vacunarse no es necesario realizar marcadores previos y recomendamos una pauta de tres dosis administradas por vía intramuscular en deltoides, con un intervalo de uno y seis meses de la primera (0, 1 y 6 meses). En personas inmunocompetentes se produce seroconversión postvacunal en un 9597\% de los vacunados (CDC, 1991b).

En nuestro medio, y dada la baja exposición por vía natural al virus de la hepatitis $A$, el porcentaje de población menor de 40 años protegida frente a él es cada día menor, por lo que recomendamos la utilización de la vacuna frente a hepatitis $\mathrm{A}$ en ese grupo de edad, administrando dos dosis por vía intramuscular en deltoides con un intervalo de 6-12 meses entre ellas.

Por último, al comenzar la vida laboral, y en caso de no estar inmunizado frente a las hepatitis A y B, se recomienda utilizar la vacuna combinada $A+B$, también administrada por vía intramuscular. La pauta vacunal consta de tres dosis $(0,1$ y 6 meses).

El resto de la Precauciones Universales también son de aplicación en odontoestomatología (McCARTHY, 1999).

\section{ESTRATEGIAS DE PREVENCIÓN. CAMPAÑA DE VACUINACIÓN}

En este contexto, el Colegio de Odontólogos y Estomatólogos de la Primera Región se planteó realizar una campaña de vacunación frente a las hepatitis A y B dirigida especialmente a sus colegiados y colaboradores y haciéndola extensiva a sus familiares.

La oferta vacunal se orientó en varias líneas:

1. Refuerzo vacunal frente a la hepatitis $B$, mediante la administración de una única dosis, en personas previamente vacunadas que no se hubieran realizado un control serológico postvacunal o éste hubiera resultado negativo.

2. Vacunación completa frente a la hepatitis B en personas no inmunizadas.

3. Vacunación completa de hepatitis A y B (vacuna combinada), en personas menores de 40 años que no tuvieran constancia de inmunización natural o adquirida frente a ninguna de las dos infecciones. 
4. Vacunación opcional frente a la hepatitis A en personas menores de 40 años.

Se han utilizado vacunas comercializadas en nuestro medio (Engerix ${ }^{\mathbb{1}}$ de $20 \mathrm{mcg}$. de $\mathrm{AgHbs}$, frente a la hepatitis B; Havrix ${ }^{\boxplus}$ de 1440 UE de antígeno viralVHA, frente a la hepatitis A; Twinrix de 720 UE. de antígeno viral-VHA y $20 \mathrm{mcg}$. de AgHBs, frente a las hepatitis A y B).

A todas las personas que participaron en la campaña se les realizó una anamnesis previa a la vacunación. Se procedió a la administración de la vacuna tras la obtención de consentimiento informado.

Las personas que, por diversos motivos, recibieron dosis de las diferentes vacunas sin seguir las pautas recomendadas no han sido consideradas en el estudio epidemiológico.

La campaña se inició en el mes de Mayo de 2000 y finalizó en Junio 2001, accediendo los interesados a la misma de forma escalonada.

\section{Aceptación - Personas vacunadas}

El número total de personas colegiadas vacunadas fue 528 odontólogos y 265 estomatólogos.

El resto de personas vacunadas se distribuyeron en 450 auxiliares, 91 higienistas, 15 protésicos y 13 diplomados universitarios en enfermería (DUEs).

Otros colectivos a los que se ha ofertado la campaña han sido estudiantes de odontología $(n=46)$, administrativos/as $(n=40)$ y familiares $(n=110)$.

En el estudio de las características de las personas potencialmente expuestas a material biológico como son las auxiliares, higienistas, protésicos, etc..., no podemos obtener conclusiones de aceptación a la campaña por desconocer la población total de los respectivos colectivos. Por esto, centraremos nuestra atención en odontólogos y estomatólogos, de los que disponemos de más información.

Vacunación en los diferentes colectivos (Tablas I y II)

\section{TABLA 1.- VACUNACIÓN FRENTE A HEPATITIS B Y HEPATITIS A+B EN COLEGIADOS Y COLABORADORES}

\begin{tabular}{|l|c|c|c|c|c|c|}
\hline & \multicolumn{3}{|c|}{ Vacuna hepatitis } & \multicolumn{3}{c|}{ Vacuna hepatitis A+B } \\
\cline { 2 - 7 } & 1 dosis & 2 dosis & 3 dosis* & 1 dosis & 2 dosis & 3 dosis* \\
\hline Odontólogos & 6 & 13 & 35 & 10 & 31 & 102 \\
\hline Estomatólogos & 1 & 15 & 19 & 9 & 10 & 31 \\
\hline Colaboradores** & 1 & 10 & 21 & 25 & 69 & 174 \\
\hline$*$ Vacunación completa. \\
** Auxiliares, higienistas, protésicos, DUEs
\end{tabular}

\section{TABLA 2.- VACUNACIÓN FRENTE A HEPATITIS A Y HEPATITIS A+B EN COLEGIADOS Y COLABORADORES}

\begin{tabular}{|c|c|c|c|c|c|}
\hline & \multicolumn{2}{|c|}{ Vacuna hepatitis $A$} & \multicolumn{3}{|c|}{ Vacuna hepatitis $A+B$} \\
\hline & 1 dosis & 2 dosis & 1 dosis & 2 dosis & 3 dosis* \\
\hline Odontólogos & 129 & 152 & 10 & 31 & 102 \\
\hline Estomatólogos & 54 & 76 & 9 & 10 & 31 \\
\hline Colaboradores ${ }^{* * *}$ & 115 & 120 & 25 & 69 & 174 \\
\hline
\end{tabular}




\section{Odontólogos}

El colectivo estudiado ascendió a 528 profesionales de un total de 2674 colegiados (19,7\%).

En relación con el tiempo trabajado, 167 odontólogos han trabajado 5 ó menos años frente al resto (361) que lo han hecho más de 5 años.

Recordaban antecedentes de accidente con material biológico 35 personas (6,6\%).

Refirieron antecedentes de alergia 67 odontólogos $(12,7 \%)$, pero ninguno de ellos lo era a componentes de las vacunas; los alergenos referidos con más frecuencia fueron pólenes.

De los 528 que aceptaron y acudieron a vacunarse, 3 presentaban antecedentes de hepatitis B, 24 de hepatitis A y 1 de hepatitis C. Estaban vacunados frente a hepatitis B 328 odontólogos (62,1\%).

Hepatitis B: Los candidatos/as a vacunación fueron 197. Se utilizó la vacuna monovalente frente a hepatitis B o la combinada frente a las hepatitis A y hepatitis $\mathrm{B}$, según los casos.

Vacuna monovalente frente a hepatitis B (B):

$\frac{\text { Incompleta }}{1 \text { dosis: } 6}-2$ dosis: $13 \quad \frac{\text { Completa }}{3 \text { dosis: } 35} \frac{\text { Total }}{54}$

Vacunación combinada frente a hepatitis $\mathrm{A}$ y hepatitis $\mathrm{B}(\mathrm{A}+\mathrm{B})$ :

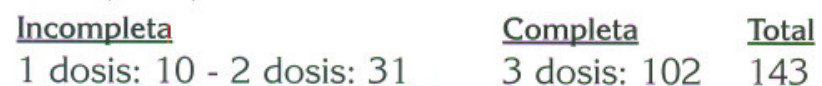

Por tanto, recibieron alguna dosis de vacuna frente a hepatitis B 197 personas (B; A+B) y completaron la vacunación 137 (69,5\%).

63 de los 328 odontólogos previamente vacunados recibieron una dosis de refuerzo $(19,2 \%)$.

Hepatitis A: Los candidatos a vacunación fueron 504.

Vacuna monovalente frente a hepatitis A (A):

Incompleta

1 dosis: 129

$\begin{array}{ll}\text { Completa } & \underline{\text { Total }} \\ 2 \text { dosis: } 152 & 281\end{array}$

Por tanto, recibieron alguna dosis de vacuna frente a hepatitis A 424 personas $(A ; A+B)$ y completaron la vacunación 254 (50,4\%).

\section{Estomatólogos}

Se estudiaron 265 profesionales de un total de 1350 colegiados $(19,6 \%)$.

En relación con el tiempo trabajado, más del $95 \%$ llevaban más de 5 años de actividad profesional.

Recordaban antecedentes de accidente con material biológico 32 personas (12,1\%).

Refirieron antecedentes de alergia 19 estomatólogos $(7,2 \%)$, pero ninguno de ellos lo era a componentes de las vacunas; la mayoría de los casos presentaban polinosis.

De los 265 que acudieron a vacunarse, siete presentaban antecedentes de hepatitis B y 18 de hepatitis A. Estaban vacunados contra la hepatitis B 173 estomatólogos (65,3\%).

Hepatitis B: Los candidatos/as a vacunación ascendieron a 85. Se utilizó la vacuna monovalente frente a hepatitis B o la combinada frente a las hepatitis A y hepatitis $B$, según los casos.

Vacuna monovalente frente a hepatitis B (B): Incompleta

1 dosis: 1 - 2 dosis: 15 Completa $\quad$ Total 3 dosis: 1935

Vacunación combinada frente a hepatitis $A$ y hepatitis $B(A+B)$ :

Incompleta

1 dosis: 9 - 2 dosis: 10

Completa $\quad$ Total 3 dosis: 3150

Por tanto, recibieron alguna dosis de vacuna frente a hepatitis B 85 personas $(B ; A+B)$ y completaron la vacunación 50 (58,8\%).

A 124 de las 173 personas previamente vacunadas frente a la hepatitis B $(71,7 \%)$, se les administró una dosis de refuerzo.

Hepatitis A: Los candidatos a vacunación fueron 247. 
Vacuna monovalente frente a hepatitis $A(A)$ : Incompleta

1 dosis: 54

Completa

2 dosis: 76

$\underline{\text { Total }}$

130

Por tanto, recibieron alguna dosis de vacuna frente a hepatitis A 180 personas $(A ; A+B)$, pero sólo completaron la vacunación 107 (43,3\%).

\section{Auxiliares, higienistas, protésicos, DUEs}

Se vacunaron un total de 569 personas: 552 mujeres y 17 hombres.

En relación con el tiempo trabajado, 250 colaboradores llevaban entre 1 y 5 años, y el resto más de 5 años.

Recordaban antecedentes de accidente con riesgo biológico 37 de ellos, la mayoría auxiliares.

Diecisiete personas refirieron antecedentes de alergia, pero ninguna de ellas lo era a componentes de las vacunas.

De los 569 colaboradoras/es que acudieron a vacunarse, 7 presentaban antecedentes de hepatitis B y 17 de hepatitis A. 254 personas estaban previamente vacunadas frente a la hepatitis $B$.

Hepatitis B: Los candidatos/as a vacunación ascendieron a 308. Se utilizó la vacuna monovalente frente a hepatitis B o la combinada frente a las hepatitis A y hepatitis $B$, según los casos.

Vacuna monovalente frente a hepatitis B (B): Incompleta Completa Total 1 dosis: 1 - 2 dosis: $10 \quad 3$ dosis: 2132

Vacunación combinada frente a hepatitis $A$ y hepatitis $B(A+B)$ :

Incompleta

1 dosis: 25 - 2 dosis: 69

Completa $\quad \underline{\text { Total }}$

3 dosis: 174268

Por tanto, recibieron alguna dosis de vacuna frente a hepatitis B 300 personas $(B ; A+B)$ y completaron la vacunación 195 (63,3\%).

Hepatitis A: Los candidatos a vacunación (menores de 40 años sin antecedentes de hepatitis A) fueron 503.

Vacuna monovalente frente a hepatitis $A(A)$ :

$\begin{array}{lll}\text { Incompleta } & \text { Completa } & \text { Total } \\ 1 \text { dosis: } 115 & 2 \text { dosis: } 120 & 235\end{array}$

Por tanto, recibieron alguna dosis de vacuna frente a hepatitis A 503 personas $(A ; A+B)$ y completaron la vacunación 294 (58,4\%).

Unida a la campaña de vacunación para sanitarios y colaboradores también ha tenido una importante aceptación la oferta a estudiantes de odontología, administrativos y familiares.

\section{Estudiantes}

Hay que recordar que los estudiantes de odontología deberían ser vacunados frente a la hepatitis B durante el primer año de sus estudios, ya que son candidatos a accidentarse con material biológico (YOUNAI, 2001). De los 46 estudiantes de odontología que acudieron a vacunarse, sólo 13 ya estaban vacunados contra la hepatitis $\mathrm{B}$ y ninguno había recibido la vacuna frente a hepatitis $A$.

Los candidatos a vacunación frente a hepatitis B fueron, por tanto, 33, pero sólo completaron la vacunación (B; A+B) 24 personas. Treinta y cinco completaron la vacunación frente a hepatitis $A(A ; A+B)$.

\section{Administrativos / as}

Las personas con funciones de administración pueden, en ocasiones, colaborar en tareas que supongan exposición a riesgo biológico, por lo que deberían estar debidamente inmunizadas. Acudieron a vacunarse 40 personas (29 mujeres y 11 hombres). 19 de ellas completaron la vacunación frente a hepatitis $B(B, A+B)$ y 32 personas lo hicieron frente a la hepatitis $A(A, A+B)$.

\section{Familiares}

Un total de 110 familiares (52 mujeres y 58 hombres, la mayoría menores de 36 años) han acudido a la campaña de vacunación del Colegio de Odontólogos y Estomatólogos. 
De los familiares que acudieron a vacunarse, 15 estaban vacunados contra la hepatitis B y 6 presentaban antecedentes de hepatitis A.

Los candidatos a vacunación frente a hepatitis B ascendieron a 95, completando la vacunación (B; A+B) $61(64,2 \%)$.

Los candidatos a vacunación frente a hepatitis A fueron 104 , completando la vacunación $(A ; A+B) 58$ $(55,8 \%)$.

\section{CONCLUSIÓN}

El estudio en estos colectivos sigue abierto y no se pueden obtener conclusiones epidemiológicas válidas, por lo que sólo constatamos la respuesta a la campaña de vacunación propuesta por el Colegio.

Por parte de los colaboradores, 569 personas han secundado la campaña, aunque llama la atención que el $36,7 \%$ no finalizó la vacuna contra la hepatitis B y el $41,6 \%$ no lo hizo con la vacunación frente a la hepatitis A.

En relación a los colegiados (odontólogos y estomatólogos) podemos afirmar que la campaña ha tenido sus efectos positivos ya que un total de 793 se han beneficiado de ella.

El 5,3\% recordaban antecedente de hepatitis A y el $1,3 \%$ de hepatitis $\mathrm{B}$; refirieron antecedente de accidente con fuente potencialmente contaminada el $8,4 \%$.

Completaron la vacunación frente a la hepatitis B el $69,5 \%$ de los odontólogos candidatos, frente a un $58,8 \%$ de los estomatólogos.

Como efectos adversos postvacunales, el 3\% de los encuestados en dosis sucesivas refirieron dolor local y el $2 \%$ eritema local. Cuatro personas comunicaron malestar general en las 48 horas siguientes a la primera dosis de vacuna frente a hepatitis A y B.

En general podemos concluir que la campaña de vacunación frente a la hepatitis $\mathrm{B}$, el riesgo más importante para odontólogos y estomatólogos, ha sido un triunfo del Colegio de Odontólogos y Estomatólogos al poner a disposición de sus colegiados la posibilidad de inmunizarse, teniendo en cuenta que estos profesionales deberían iniciar su vida laboral debidamente protegidos.

Sería deseable conseguir coberturas elevadas de vacunación frente a la hepatitis B semejantes a otros colectivos de dentistas (GORE, 1994; VIGNARAJAH, 1998), sin olvidar la posibilidad de inmunizarse contra la hepatitis A con la utilización de una vacuna combinada.

Por último recordar la obligación de informar y ofertar la inmunización, cuando esté indicada, a los colaboradores (Ministerio de Sanidad y Consumo, 2001).

\section{BIBLIOGRAFÍA}

- Alter MJ, Hadler SC, Margolis HS y cols. The changing epidemiology of hepatitis $B$ in the United States. JAMA 1990; 263: 1218-22.

- Ashkenazi M, Chodik G, Littner M y cols. The presence of hepatitis A antibodies in dental workers. A seroepidemiology study. J Am Dent Assoc 2001; 132: 492-8.

- Centers for Disease Control and Prevention. Update: Universal precautions for prevention of transmisssion of Human Immunodeficiency Virus, Hepatitis B virus, and other blood-borne Pathogens in health-care settings. MMWR 1988; 37: 377-82, 387-8.

- Centers for Disease Control and Prevention. Transmission of HIV infection during invasive dental procedures - Florida. MMWR 1991; 40: 21-7, 33.

- Centers for Disease Control and Prevention. A comprehensive strategy for eliminating transmission in the United States through universal childhood vaccination. MMWR 1991b; 40 (RR-13): 1-25.

- Centers for Disease Control and Prevention. Practical Infection Control in the Dental Office. U.S. Departament Health $\&$ Human Services. C.D.C. 1993. 
- Centers for Disease Control and Prevention. Recommended Infection-Control Practices for Dentristry, 1993. MMWR 1993b; 41 (RR-8): 1-12.

- Chen M, Yun ZB, Sallberg My cols. Detection of hepatitis $C$ virus RNA in the cell fraction of saliva before and after oral surgery. J Med Virol 1995; 45: 223-6.

- Ciesielski C, Marianos D, Ou CY y cols. Transmission of human immunodeficiency virus in a dental practice. Ann Intern Med 1992; 116: 798-805.

- Cleveland JL, Siew C, Lockwood SA y cols. Factors associated with hepatitis B vaccine response among dentists. J Dent Res 1994; 73: 1029-35.

- Cleveland JL, Siew C, Lockwood SA y cols. Hepatitis B vaccination and infection among U.S. dentists, 1983-1992. J Am Dent Assoc 1996; 127: 1385-90.

- Cleveland JL, Gooch BF, Schearer BG y cols. Risk and prevention of hepatitis $C$ virus infection. Implications for dentistry. J Am Dent Assoc 1999; 130: 641-7.

- de Juanes JR. Hepatitis B: presente y futuro de la vacunación. Hepatol Clin 1994; 8: 89-97.

- de Juanes, JR, Arrazola MP, Domçinguez V y cols. Problemática de los agentes biológicos en la práctica odontológica. Normas en la clínica diaria. Avances en Odontología 1996; 12 (4):277-92.

- Gillcrist JA. Hepatitis viruses A, B, C, D, E and G: implications for dental personnel. J Am Dent Assoc 1999; 130: 509-20.

- Gore SM, Felix DH, Bird AG y cols. Occupational risk and precautions related to HIV infection among dentists in the Lothian region of Scotland. J Infect 1994; 28: 209-22.

- Goupil MT. Occupational health and safety emergencies. Dent Clin North Am 1995; 39: 637-47.

- Hoofnagle JH. Chronic hepatitis B. N Engl J Med 1990; 323: 337-9.
- McCarthy GM, Koval JJ, MacDonald JK. Compliance with recommended inefction control procedures among Canadian dentist: results of a national survey. Am J Infect Control 1999; 27: 377 84.

- Ministerio de Sanidad y Consumo. Plan Nacional sobre el SIDA. Prevención de la infección por virus de transmisión sanguínea (VIH, VHB y VHC) en odontoestomatología. Madrid, 2001.

- Palenik CJ. Human hepatitis viruses. J Dent Technol 1999; 16: 20-3.

- Reichart PA. AIDS and hepatitis: a problem for the dental team. Int Dent J 1994; 44: 49-54.

- Resnick RH, Koff R. Hepatitis C-related hepatocellular carcinoma. Prevalence and significance. Arch Intern Med 1993; 153: 1672-7.

- Sallie R, King R, Silva E y cols. Community prevalence of hepatitis $\mathrm{C}$ viraemia: a polymerase chain reaction study. J Med Virol 1994; 43: 111-4.

- Vignarajah S, Eastmond VH, Ashraph A y cols. An assesment of cross-infection control procedures among English-speaking Caribbean general dental practitioners. A regional preliminary study. Int Dent J 1998; 48: 67-76.

- Wolff M, Hidalgo S. Exposición accidental del personal de salud a sangre y líquidos biológicos de pacientes. Rev Med Chil 1992; 120: 1247-53.

- Younai FS, Murphy DC, Kotelchuck D. Occupational exposures to blood in a dental teaching environment: results of a ten-year surveillance study. J Dent Educ 2001; 65: 436-48.

\section{CORRESPONDENCIA}

Prof. J.R. de Juanes Pardo.

Servicio de Medicina Preventiva. Ctra. de Andalucía Km. 5,4. 28041 Madrid.

Tel : 91-3908026

e-mail: jrj@medynet.com 